\title{
Design and Implementation of Real Time Health Monitoring Care Unit Using IOT
}

\author{
A.V.Khapre ${ }^{1}$, Akshay R. Gotmare ${ }^{2}$, Bhushan C. Parate ${ }^{3}$, Dnyaneshwar I. Belkhede ${ }^{4}$, \\ Gaurao C. Parate \\ ${ }^{1}$ Assistant professor, ${ }^{2,3,4,5}$ UG Student \\ Department of ElectricalEngineering, Nagpur Institute of Technology, Nagpur, India,440026
}

\begin{abstract}
The body sensor network automation is a specific core technology of IoT domain in healthcare technique, where a sick person can be tracked of his/her health using a collection of body parameters with the use of minute powered and flimsy wireless sensor nodes. The largely imperative intent of the endeavor was to craft a distant health care system. It's comprised of three focal sections. The earliest section being, revealing of patient's vitals via sensors, second for conveyance information to obscure storage and the third bit was providing the detect information for secluded performance. Secluded performance of the information enables a practitioner or custodian to scrutinize a patient's well-being progress missing from sanatorium property.

The topical advancement in expertise and the accessibility of the Internet make it feasible to hook up a variety of campaign that can converse with each other and allocate data. Internet of Things (IoT) can be defined as the wireless unification of campaign which are allied to each other to allocate information and data in bid to commune and construct new information so as to trace and analyze it for hope exploit. The anticipated sculpt enable user to perk up health concurrent risk and shrink healthcare expenditure by collect, footage, analyzing and partaking huge information stream in genuine instance and ably. The notion of this venture come so to ease the nuisance of serene to break to practitioner each occasion he necessitate to ensure his blood pressure, pulse rate, temperature and so forth.
\end{abstract}

Keywords- Internet of Things (IoT), Arduino Uno, Thingspeak

\section{I- INTRODUCTION}

D ue to the hi-tech advance in the present day, there is huge assortment successively feeler construal imperative cipher such as blood pressure, heart rate monitor, counting electrocardiograms, which tolerate patients to take their crucial cryptogram day by day. The interpretations which are engaged on a daily basis are remitting to doctors and they will counsel the linctus and drills routine that allow them to progress the worth of life and surmount such disease. The internet of things applied to the heed and monitoring of patients. This manuscript proposes a vigour monitoring system which is capable of detecting manifold stricture of our body such as blood pressure, temperature, heart rate.An incessant record of body can be used to distinguish an illness in more competent way. Anticipated system consists of a temperature, blood pressure, pulse sensor, and Arduino system is competent of measure pulse rate, temperature and blood pressure of infant to ripened person. Low cost of contrivance assists to grant apposite handy inaccessible base effectual heart rate monitoring system.

Now, Internet of Things (IoT) has become one of the most authoritative communication paradigms of the 21 th century. It is chiefly a compilation of low-power and glare credibility wireless sensor nodes that are used to scrutinize the human body function and contiguous environment. Since BSN nodes are used to accumulate perceptive (life-critical) information and may manoeuvre in antagonistic environments. consequently, they entail austere refuge mechanism to prevent cruel dealings with the system.

\section{PROPOSED DESIGN}


The universal intend of IoT application can be partition into three Layers: the detecting layer, the convey stratum and the application layer. In the Detecting layer to appraise the body temperature we Utilized DS18B20 body water contravene body temperature feeler. To assess the heart beat Rate of the patient by utilizing pulse sensor amped is utilized. It has its own fastidious an envisage to depiction the heart beat waveform, BPM.It gives the data about whether the patient is sitting, resting, sleeping, walking, jumping or frontbend and diverse circumstances. To recognize the Blood pressure and Pulse rate we utilized sunrom BP sensor. This Bio data from the sensors is tartan unremittingly in Arduino utilizing the consecutive scrutinize. In the Transport layer we remit the information to the cloud by utilizing the Arduino with the aid of Wi-Fi Module ESP8266.

Here we make use of the open cloud server i.e. "Thingspeak" to build it easily reached in cloud, with the end target that the data can be go to anywhere on the planet. Here in the Thingspeak server we make an own account and we make a our private channel by the name of our collage. So by making the own channel we get a remarkable id and API-key to compose the information to the cloud. This API key is use while programming in Arduino with the purpose that the information is put in the server and at the application layer we can recover the information by the use of one of a kind API key to such an extent that the information can be obtained from the server.

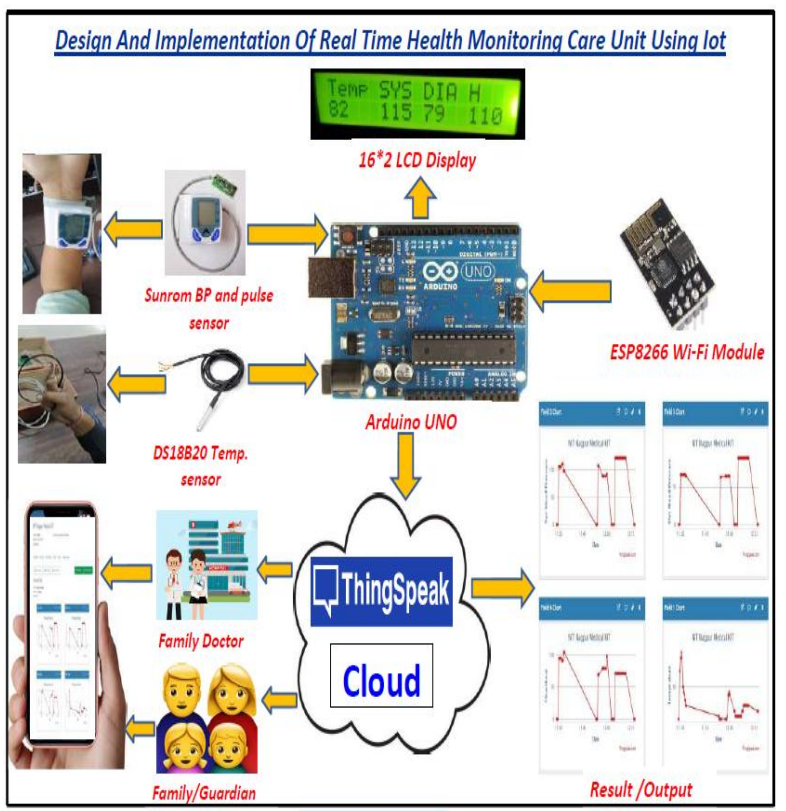

Fig-1: Proposed system

\section{PATIENT MONITORING ELEMENTS}

\section{A. Body Temperature Sensor}

The DS18B20 is the one of the best reasonable sensor for measuring the human body temperature (exact to $\pm 0.5^{\circ}$ Centigrate over the extent of $-55^{\circ} \mathrm{C}$ to $+125^{\circ} \mathrm{C}$ ) .We can put these sensors at different spots of the human body like on forehead, under arms and even under the tongue on account of its waterproof. The DS18B20 has three wires i.e. VCC, GND, DATA are mounted in a solitary wire. The DS18B20 uses Maxims exclusive 1-Wire bus protocol that implements bus communication using one control signal. The control line requires a weak pullup resistor since all devices are linked to the bus via a 3-state or open-drain port (the DQ pin in the case of the DS18B20). In this bus system, the microprocessor (the master device) identifies and addresses devices on the bus using each devices unique 64-bit code. Because each device has a unique code, the number of devices that can be addressed on one bus is virtually unlimited. The 1-Wire bus protocol, including detailed explanations of the commands and time slots, is covered in the 1-Wire Bus System section. Another feature of the DS18B20 is the ability to operate without an external power supply. The core functionality of the DS18B20 is its direct-to digital temperature sensor. The resolution of the temperature sensor is user-configurable to $9,10,11$, or 12 bits, corresponding to increments of $0.5 \mathrm{C}, 0.25 \mathrm{C}, 0.125 \mathrm{C}$, and $0.0625 \mathrm{C}$, respectively. The default resolution at power-up is 12-bit.

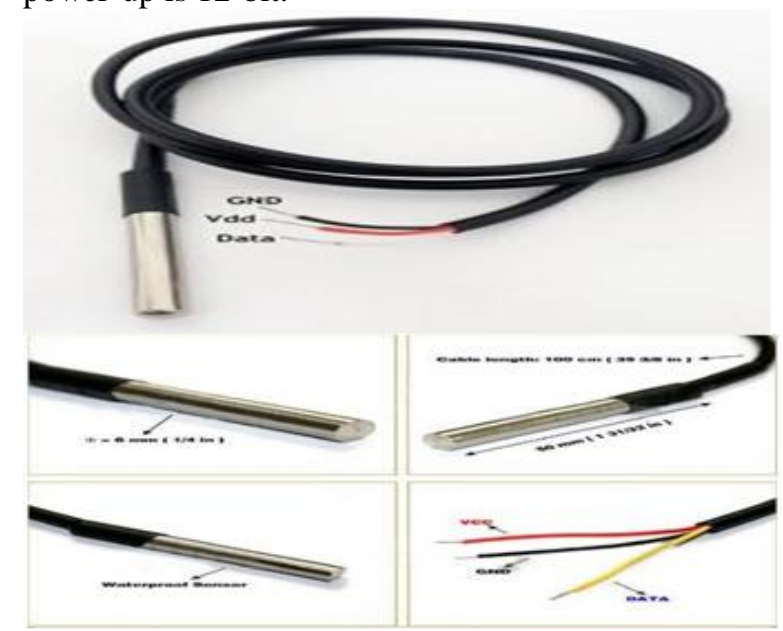

Fig-2:DS18B20 Temperature Sensor

\begin{tabular}{|l|l|}
\hline $\begin{array}{l}\text { Power Supply } \\
\text { Range: }\end{array}$ & $3.0 \mathrm{~V}$ to $5.5 \mathrm{~V}$ \\
\hline $\begin{array}{l}\text { Operating } \\
\text { Temperature } \\
\text { Range: }\end{array}$ & $\begin{array}{l}-55^{\circ} \mathrm{C} \text { to }+125^{\circ} \mathrm{C}(-67 \mathrm{~F} \text { to } \\
+257 \mathrm{~F})\end{array}$ \\
\hline Storage & $-55^{\circ} \mathrm{C}$ to $+125^{\circ} \mathrm{C}(-67 \mathrm{~F}$ to \\
\hline
\end{tabular}




\begin{tabular}{|l|l|}
\hline $\begin{array}{l}\text { Temperature } \\
\text { Range }\end{array}$ & $+257 \mathrm{~F}$ \\
\hline $\begin{array}{l}\text { Accuracy over the } \\
\text { range of } \\
-10 \mathrm{C} \text { to }+85 \mathrm{C}\end{array}$ & $\pm 0.5^{0} \mathrm{c}$ \\
\hline Size of Sheath & $6^{* 50 \mathrm{~mm}}$ \\
\hline Connector: & RJ11/RJ12, 3P-2510, USB. \\
\hline Pin Definition: & $\begin{array}{l}\text { RED: VCC } \\
\text { YELLOW: DATA } \\
\text { BLACK: GND }\end{array}$ \\
\hline Cable length: & $\begin{array}{l}1 \text { meter, } 2 \mathrm{~m}, 3 \mathrm{~m}, 4 \mathrm{~m} \text { are } \\
\text { Available }\end{array}$ \\
\hline
\end{tabular}

If the DS18B20 is configured for 12-bit resolution, all bits in the temperature register will contain valid data. For 11-bit resolution, bit 0 is undefined. For 10-bit resolution, bits 1 and 0 are undefined, and for 9bit resolution bits 2,1 , and 0 are undefined. Table 1 gives examples of digital output data and the corresponding temperature reading for 12-bit resolution conversions.

\section{Applications:}

Since each DS18B20 contains an ideal silicon serial number, more numerous DS18B20s can exist on the same 1Wire transport. This considers putting temperature sensors in a wide range of spots. Applications where this component is valuable incorporate HVAC natural controls, detecting temperatures inside structures, gear or apparatus, and process checking and control.

\section{B. Sunrom Blood Pressure and Pulse Sensor}

This sensor is has Intelligent automatic compression and decompression system which makes it easy to operate with switching button to start the measurements with Large-scale digital liquid crystal display screen.

It plant on $200 \mathrm{~mA}$ regulated current from $5 \mathrm{v}$ power supply. Its Output Format is Serial Data at 9600 baud rate( 8 bits data, No parity, 1 stop bits). Outputs three parameters in ASCII. Blood pressure is the fretfulness of the blood in the arteries as it is pumped around the body by the heart.

When your heart beats, it contracts and pushes blood through the arteries to the respite of your body. This oblige creates pressure on the arteries. Blood pressure is recorded as two figures the systolic anxiety (as the heart beats) over the diastolic anxiety (as the heart relaxes between beats). The unit which course this is called Sphygmomanometer. Monitoring blood pressure at home is imperative for many people, chiefly if you have high blood pressure. Blood pressure does not reside the same all the instant. It changes to convention your body's wants. It is pretentious by assorted factors counting body position, breathing or emotional state, exercise and sleep. It is best to appraise blood pressure when you are relaxed and sitting or lying down.

High blood pressure (hypertension) can pilot to solemn nuisance like heart attack, stroke or kidney disease. High blood pressure (hypertension) can lead to grave exertion like heart attack, stroke or kidney disease. High blood pressure usually does not have any symptoms, so you need to have your blood pressure chequered habitually.

\section{Features:}

1. Intelligent involuntary compression and decompression

2. Easy to manoeuvre, switching button to start measuring

3. 60 store groups reminiscence measurements

4. Can interpret single or all measures

5. 3 minutes involuntary supremacy cutback appliance

6. Intelligent device debugging, routine clout to perceive

7. Local tests for : wrist fringe as $135-195 \mathrm{~mm}$

8. Large-scale digital liquid crystal flaunt screen, Easy to Read Display

9. Fully Automatic, Clinical Accuracy, High-accuracy

10. Power by External $+5 \mathrm{~V}$ DC

11. Serial output data for external circuit processing or display.

\section{Specification:}

1. Working Voltage: $+5 \mathrm{~V}, 200 \mathrm{~mA}$ regulated

2. Output Format :Serial Data at 9600 baud rate( 8 bits data, No parity, 1 stop bits). Outputs three parameters inASCII.

3. Sensing unit wire length is 2 meters

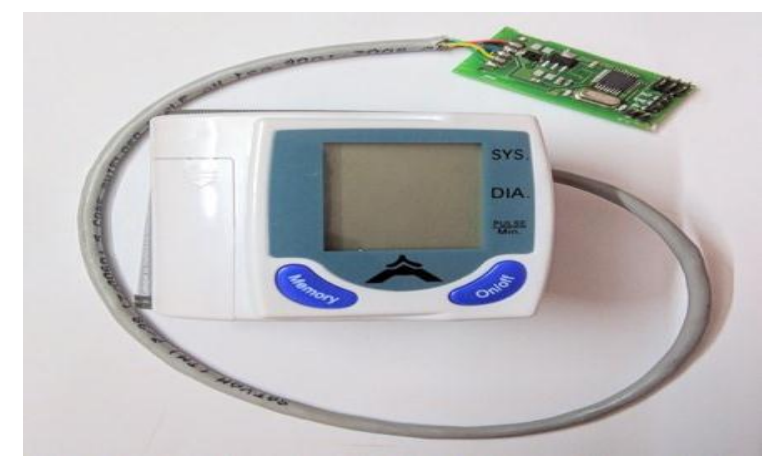

Fig-3: Sunrome BP and Pulse Rate Sensor

\section{Sensor Pinouts:}

1. TX-OUT $=$ Transmit output. Output serial data of $3 \mathrm{~V}$ logic level usually connected to RXD pin of microcontroller/RS232/USB-UART.

2. $+5 \mathrm{~V}=$ Regulated $5 \mathrm{~V}$ supply input. 
3. GND = Board Common Ground. Classification of blood pressure for adults (18 years and older)

High blood pressure (hypertension) can lead to serious problems like heart attack, stroke or kidney disease. High blood pressure usually does not have any symptoms, so you need to have your blood pressure checked regularly.

Classification of blood pressure for adults (18 years and older)

\begin{tabular}{|c|c|c|}
\hline & $\begin{array}{c}\text { Systolic }(\mathbf{m m} \\
\mathbf{H g})\end{array}$ & $\begin{array}{c}\text { Diastolic } \\
(\mathbf{m m} \text { Hg) }\end{array}$ \\
\hline Hypotension & $<90$ & $<60$ \\
\hline Desired & $90-119$ & $60-79$ \\
\hline Prehypertension & $120-139$ & $80-89$ \\
\hline Stage 1 Hypertension & $140-159$ & $90-99$ \\
\hline Stage 2 Hypertension & $160-179$ & $100-109$ \\
\hline Hypertensive Crisis & $\geq 180$ & $\geq 110$ \\
\hline
\end{tabular}

\section{Wi-Fi Module ESP8266}

The ESP8266 is low-cost Wi-Fi microchip with full TCP/IP stack and microcontroller competence fashioned by Shanghai-based Chinese manufacturer, Express if Systems. The chip first came to the thought of western makers in August 2014 with the ESP-01 module, made by a third-party firm, Ai-Thinker.

The ESP8266 WiFi Module is a self-restricted SOC with incorporated TCP/IP protocol stack that can give any microcontroller entrée to your WiFi network. The ESP8266 is accomplished of either hosting an application or offloading all Wi-Fi networking functions from another claim workstation.

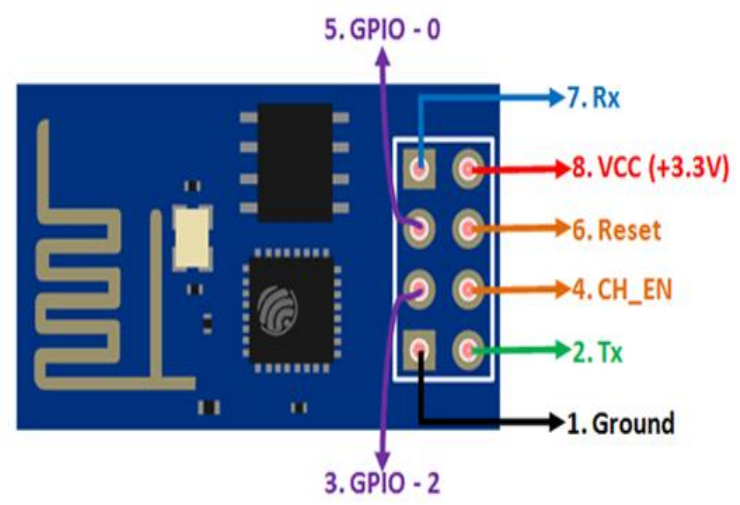

Fig-4-ESP8266 Wi-Fi Module
Each ESP8266 module comes pre-programmed with an AT command set firmware, meaning, you can merely clip this up to your Arduino contrivance Mand get about as much WiFi-ability as a WiFi shelter offers . The ESP8266 module is an exceedingly cost effectual timber with a colossal, and still budding, community.

\section{ESP8266 Module Pin Description:}

1. 3V3: - 3.3 V Power Pin.

2. GND: - Ground Pin.

3. RST: - Active Low Reset Pin.

4. EN: - Active High Enable Pin.

5. TX: - Serial Transmit Pin of UART.

6. RX: - Serial Receive Pin of UART.

7. GPIO0 ; GPIO2: - General Purpose I/O Pins.

\section{Features:}

Processor: L106 32-bit RISC microprocessor core based on the Tensilica

Xtensa Diamond Standard 106Micro running at 80 $\mathrm{MHz}$

1] Memory:

1. $32 \mathrm{KiB}$ instruction RAM

2. $32 \mathrm{KiB}$ instruction cache RAM

3. $80 \mathrm{KiB}$ user-data RAM

4. $16 \mathrm{KiB}$ ETS system-data RAM

2] External QSPI flash: up to $16 \mathrm{MiB}$ is supported (512 $\mathrm{KiB}$ to

$4 \mathrm{MiB}$ typically included)

3] IEEE $802.11 \mathrm{~b} / \mathrm{g} / \mathrm{n} \mathrm{Wi}-\mathrm{Fi}$

\section{SYSTEM IMPLEMENTATION}

Here we exploit the information that has been poised to the cloud to screen the patient's wellbeing stipulation. While making a channel in the Thingspeak server we make idiosyncratic channels for our exploitation, for occasion, we make a channel which incorporates the information of unambiguous patient, and another channel for the other and as so on.

In every personage channel we make sub fields for various wellbeing parameters counting Blood pressure, Temperature, pulse rate. We can scrutinize the intact information of the patient in a graphical view. Indeed, even we can exploit this information for exploration in MATLAB for further additional operations.

As we utilize the Thingspeak open cloud server to screen the information, every last esteem that has been gotten from the sensor sited at the patients end is exchanged candidly for every 15 seconds of customary intervals.

With the end ambition that any sudden changes in the patient's wellbeing stipulation can be pragmatic rapidly and punctual can be made to the personage establishment We had a Thingspeak Android application to such an extent that the patient information can be observed independently in a 
cosseted way with the assistance of the unusual APIkey.

Indeed, even this information is put away for a lifetime and can be gotten to whenever consequently can be utilized for future references.

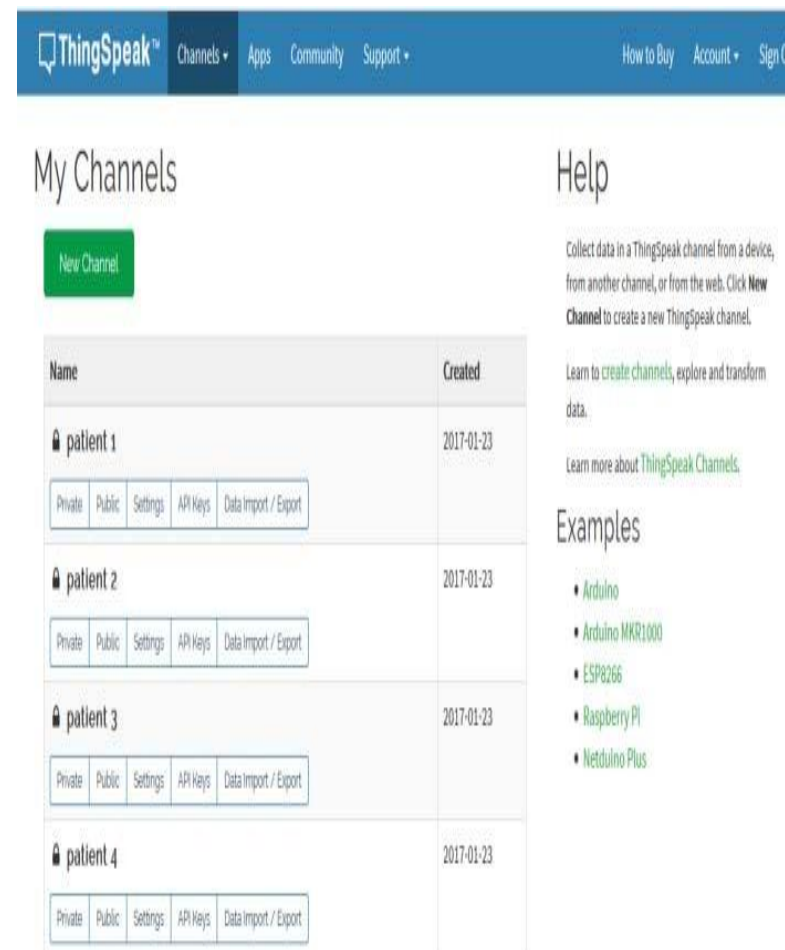

Fig-5: Thingspeak cloud with patient channels

As showed in the above figure, we have made four inimitable channels and named them as patient $1,2,3,4$. There are sundry sub-sections in every channel, for example, private, public.

Where are the concealed can be empowered to such an extent that the information can be gotten to just by the approved people who comprises of the incomparable API-Key and whereas out in the public everybody with the channel-ID can access to the information. Also, we can Import/Export the Data in excel sheet for nittygritty discernment of the patient.

\section{V- RESULTS}

The graphical representation of the information of the patient-1 is demonstrated i.e. body temperature, Heartbeat, blood pressure are as showed up in the fig which is observed by signing into the Thingspeak server through a personal computer which has Time in $\mathrm{x}$-axis and the parameter in $\mathrm{y}$-axis.

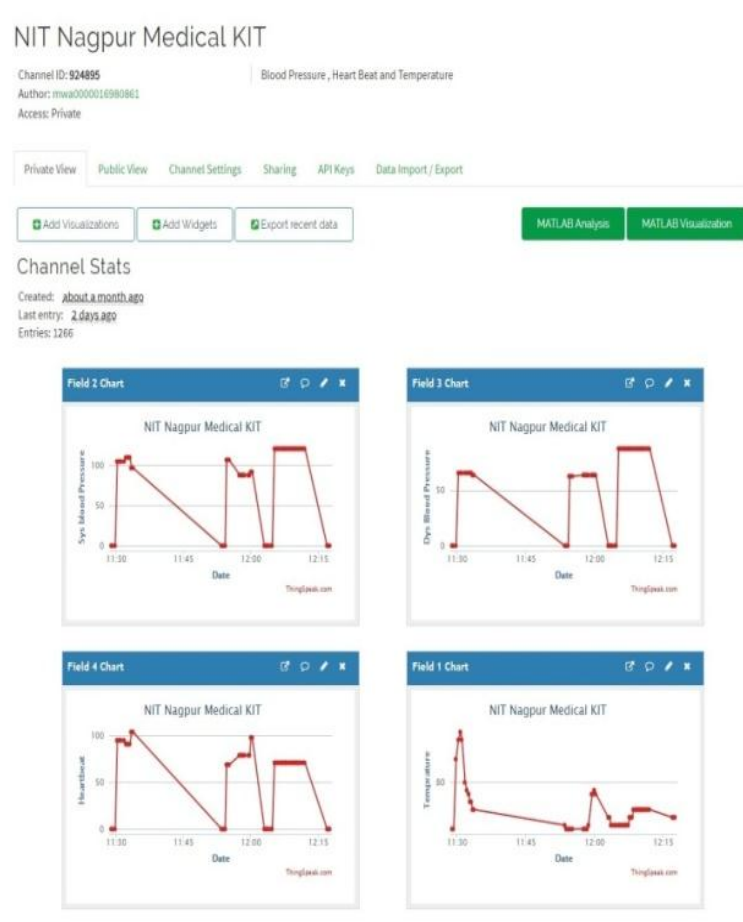

Fig-6: Thingspeak cloud with patient channels

\section{VI- CONCLUSION}

The proposed system collects the health status of a patient by connecting the different sensors to the body. The collected data is displayed on LCD and also stored in IoT server. The doctor can view the patients health condition by login into the web portal. From the evaluation and the result obtained from analysis, the system is better for patients and the doctor to improve their patients' medical evaluation.

\section{FUTURE SCOPE}

The system can be extended by adding more features to the mobile application like linking the ambulance services, leading doctor's list and their specialties, hospitals and their special facilities etc., Doctors can create awareness about diseases and their symptoms through the mobile application.

\section{REFERENCES}

[1] P. Gope and T. Hwang, BSN-Care: A Secure IoT Based Modern Healthcare System Using Body Sensor Network, IEEE Sensors Journal, vol.16, no. 5, pp. 13681376, 2016.

[2] S. Sarkar and S. Misra, From Micro to Nano: The Evolution of Wireless Sensor-Based Health Care, IEEE Pulse,// vol. 7, no. 1, pp. 2125, 2016.

[3] D. V. Dimitrov, Medical Internet of Things and Big Data in Healthcare, Healthcare Informatics Research, vol. 22, no. 3, pp. 156163, 72016. 
[4] G. Wolgast, C. Ehrenborg, A. Israelsson, J. Helander, E. Johansson, and H. Manefjord, Wireless Body Area Network for Heart Attack Detection [Education Corner], IEEE Antennas and Propagation Magazine, vol.58, no. 5, pp. 8492, 2016.

[5] S. Milici, J. Lorenzo, A. Lazaro, R. Villarino, and D. Girbau, Wireless Breathing Sensor Based on Wearable Modulated Frequency Selective Surface, IEEE Sensors Journal, vol. PP, no. 99, p. 1, 2016.

[6] H. El-Sayed and G. Thandavarayan, Congestion Detection and Propagation in Urban Areas Using Histogram Models, IEEE Internet of Things Journal, vol. PP, no. 99, p. 1, 2017.40

[7] T. C. Arcadius, B. Gao, G. Tian, and Y. Yan, Structural Health Monitoring Framework Based on Internet of Things: A Survey, IEEE Internet of Things Journal, vol. PP, no. 99, p. 1,2017. 